\title{
Tutorial: Perl, a psychologically efficient reformatting language
}

\author{
ALAN SCHWARTZ \\ University of Illinois, Chicago, Illinois
}

\begin{abstract}
Psychologists are often faced with the need to manipulate one or more files, either to modify the format or to extract specific information. Traditionally, these manipulations have been performed using programming languages or statistical software, but such solutions are often expensive, platform dependent, or limited in their ability to handle both numerical and textual data. This tutorial introduces the perl programming language, a free, platform-independent language that excels at pattern matching and text processing but that is also numerically capable. A running example illustrates an application of perl to psychological data.
\end{abstract}

Psychologists are often faced with the need to manipulate one or more files, either to modify the format or to extract specific information. For example, reaction time measurements, demographic information, and a transcribed think-aloud protocol might be recorded in one file for each subject, and a variety of analyses might require extracting and summarizing data of different types.

Traditionally, these manipulations have been performed using programming languages such as FORTRAN, Pascal, or C, or statistical software such as SAS (SAS Institute, 1996). However, these solutions suffer from a number of limitations. Most of these packages have strong numerical features but are poor at handling text. Software may be expensive or difficult to find for a particular computing platform.

This tutorial introduces the perl programming language, a free, platform-independent language that excels at pattern matching and text processing. A running example illustrates an application of perl to psychological data.

\section{WHAT IS PERL?}

Perl, the "practical extraction and reporting language,"' was created by Larry Wall (1986) as an alternative to two venerable UNIX-based text-processing programs, awk and sed. Its current incarnation, perl 5.005, is a full-featured programming language that includes mathematical functions, networking capabilities, a builtin debugger, object-oriented programming, and other sophisticated tools. Despite its power, perl's hallmark has been its intuitiveness and ease of use: "Perl is designed to make the easy jobs easy without making the hard jobs impossible" (Wall, Christiansen, \& Schwartz, 1996). It

Alan Cooke provided helpful comments on a draft of this article. Correspondence should be addressed to A. Schwartz, Department of Medical Education (m/c 591), $808 \mathrm{~S}$. Wood Street, $986 \mathrm{CME}$, University of Illinois, Chicago, IL $60612-7309$ (e-mail: alansz@uic.edu). has become very popular with UNIX system administrators, webmasters, and others who regularly process text files.

Many tutorial and reference books about perl are available. Perl is distributed with an extensive on-line manual that documents all aspects of the language. O'Reilly and Associates publishes a series of books that are generally considered to be the canonical perl presentations. Schwartz and Christiansen's (1997) Learning Perl is the standard beginner's tutorial and requires little background in programming. Wall et al.'s (1996) Programming Perl is the definitive reference guide to perl and forms a logical second book for users seeking to harness features beyond those described in Schwartz and Christiansen. Srinivasan's (1997) Advanced Perl Programming covers the more powerful features of the language, including data structures, object-oriented programming, networking, and graphical interface design. Other publishers have also produced books on learning and using perl.

Perl is available for UNIX workstations, PCs, Macintoshes, and other platforms; version numbering and distribution format varies somewhat from platform to platform. It is free software and can be downloaded from the World-Wide Web at http://www.perl.com/CPAN/. Many UNIX systems are now distributed with perl already installed.

Perl is an interpreted language, like SAS, rather than a compiled language, like FORTRAN. Perl programs (also called "scripts") are processed by the perl interpreter line by line. Following is a sample from a perl program that prints a countdown from 10 to 0 using a "for" loop (line numbers are used for reference only and do not appear in the program itself):

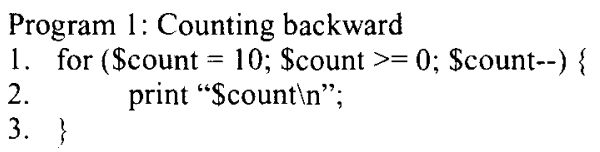

In Program 1, the "for" statement sets up a loop in which the scalar variable \$count is initially 10. The loop 
will continue as long as \$count is greater than or equal to 0 , and at each iteration, \$count will be decremented by 1 (\$count--). Line 2 prints the value of \$count followed by a newline character ("In").

The syntax is (intentionally) very similar to the C programming language. Blocks of statements are enclosed in curly braces, and statements end in semicolons. In fact, someone more familiar with $\mathrm{C}$ might instead write the program like this:

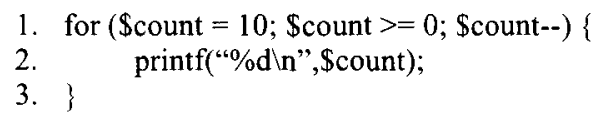

This is also acceptable perl.

\section{VARIABLES}

Perl understands three kinds of simple variables. Scalar variables contain numbers or strings and are indicated by a $\$$ in front of the variable name (e.g., $\$$ count, as above). When they contain numbers, common mathematical functions and operators are available to manipulate them:

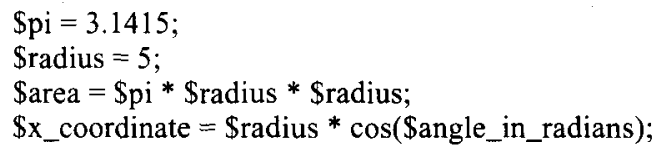

When scalar variables contain strings, string functions and operators can be applied:

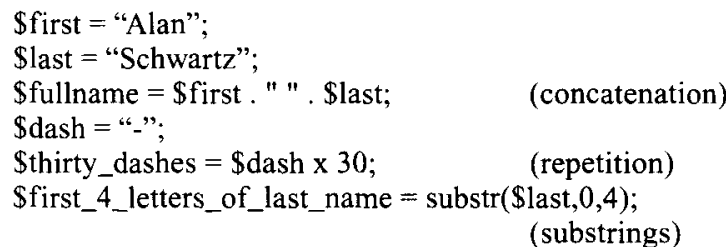

List variables are similar to arrays in other programming languages. They contain ordered lists of scalars, indexed by numerical position in the list, and are indicated by @ in front of the variable name (e.g., @ favorite_things). Individual list items, however, are scalars and are prefixed with $\mathrm{S}$ :

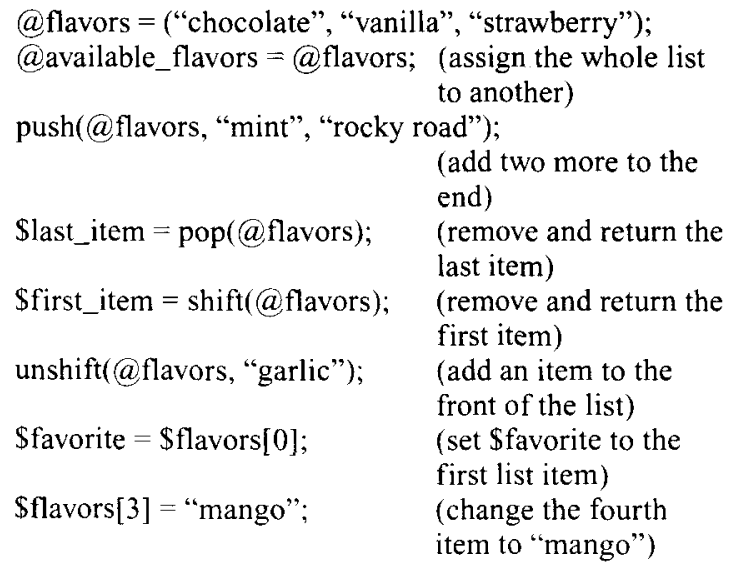

As the sample code shows, a list of $N$ elements is indexed with numbers that range from 0 to $N-1$. List variables are an intuitive way to represent an ordered series of data points.

Associative arrays (or "hashes") are unordered collections of key-value pairs and are indicated by a $\%$ in front of the variable name. They are an ideal way to associate data with a meaningful identifier (e.g., a stimulus or subject name):

$$
\begin{aligned}
& \% \text { first_names }=(\text { "Clinton" }=>\text { "Bill", "Monroe" } \Rightarrow \\
& \text { "Marilyn", "Jordan" } \Rightarrow \text { "June" ); } \\
& \text { Spresident }=\text { \$first_name }\{\text { "Clinton" }\} ; \text { (look up Clinton's } \\
& \text { first name) } \\
& \text { \$first_name }\{\text { "Freud"\} = "Anna"; } \quad \text { (add a new pair to } \\
& \text { the associative } \\
& \text { array) } \\
& \text { delete(Sfirst_name\{"Monroe" }\} \text { ); } \quad \text { (remove a pair) }
\end{aligned}
$$

\section{MORE THAN ONE WAY}

The perl motto is, "There's more than one way to do it." Following is Program 1 rewritten using the "foreach" statement, which loops over a list of values:

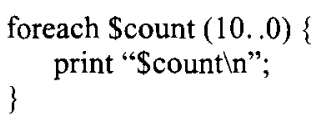

Scount takes on the values from 10 to 0 , in order. To count with English words, we might instead write:

$$
\begin{aligned}
& \text { foreach \$count ("ten", “nine", "eight", “seven", "six", } \\
& \text { "five", "four", "three", "two", "one", "zero") ( }
\end{aligned}
$$

or, for clarity, we could use an array variable to hold the numbers:

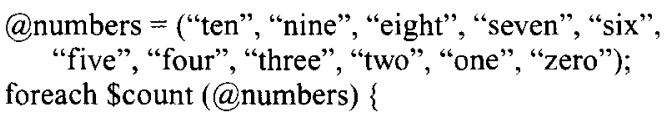

\section{WRITING AND RUNNING PERL SCRIPTS}

To work in perl, all you need is an editor and a perl interpreter. Because perl scripts are text files, any program that can edit text files can be used to write perl scripts. This includes simple text editors (e.g., Windows Notepad or UNIX emacs) and fancy word processors (e.g., Microsoft Word).

How to run a perl script varies somewhat depending on the type of operating system that the computer uses, but most computers can run perl scripts by typing

perl [perl-options] script-filename [arguments]

or by running the perl interpreter in some other fashion (e.g., double-clicking a "perl" icon) and giving it the filename of the script and a list of arguments. These might be the names of files that you would like to process.

The optional perl options control the way the interpreter runs the script. Commonly used options include 
"-cw" (check the script for errors but do not actually run it) and "-d" (debug the script using the perl debugger).

\section{REAL-WORLD EXAMPLES}

To illustrate the power of perl, imagine a study that measures subjects' reaction times (RTs) in milliseconds in each of three conditions (RT1, RT2, and RT3), as well as their age in years (AGE) and a think-aloud protocol during a jigsaw puzzle task. Each subject's data on all of the tasks are entered into a text file for that subject that looks like this:

RT 1: 300

RT2: 250

RT3: 200

AGE: 19

PROTOCOL:

First I tried to find the corners. Then I put together the edges of the puzzle. Finally, I filled in the center by using the picture on the front of the box.

\section{EXAMPLE 1: PATTERN MATCHING}

Perl's pattern-matching capabilities are based on regular expressions, a powerful and flexible way to describe patterns. A regular expression can be as simple as "the letter " $a$ ' anywhere in the string" or as complex as "one or more capitalized words, a comma, one or more spaces, 2 uppercase letters, one or more spaces, and 5 digits, optionally followed by a hyphen and 4 more digits," which might describe the last line of an address.

Following is an example of how one might extract the age from a single file given as an argument to the script, followed by a step-by-step explanation.

1. \$filename=shift $(@$ ARGV);

2. \# If we can't open the file, quit and complain

3. open(IN,\$filename) or die "I couldn't open \$filenameln";

4. while $(<\mathrm{IN}>)$ \{

5. chop;

6. print "The age is: $\$ 1 \backslash n$ " if $/ \wedge$ AGE: $\left(*^{*}\right) /$

7. $\}$

8. $\operatorname{close}(\mathrm{IN})$

Arguments to a perl program are accessible to the program as the list@ARGV. The shift() function removes the first element of a list and returns it. In line 1, \$filename is set to the first element of @ARGV, which should be the filename given as an argument to the program.

Line 2 illustrates a comment in perl. Comments begin with a pound sign and may appear anywhere.

In line 3, the open() function opens a file handle called IN and associates it with \$filename. Or, if the file can't be opened, the program "dies"-- stops running and prints an error message. As this line illustrates, perl programs can often be read aloud: "open Sfilename or die."
The file is opened for reading; to open a file for writing, the statement open(OUT, “>\$filename") is used.

Line 4 introduces a "while" loop and uses the file handle input operator $<>$, which returns the next line from the file or the special value "undef" at the end of the file. The lines are stored in a temporary buffer. The "while" loop will continue while there are lines to be read from the file.

Line 5 "chops" (removes) the newline character that appears at the end of each line that is read in from the file. This is a common operation, because it is rarely useful to work with the newline characters.

Line 6 performs regular expression pattern matching using the // operator. It can be read as "print some text if we are on a line that begins with AGE:"' In the regular expression enclosed in the slashes, the caret indicates that the match must begin at the beginning of the line (rather than anywhere in the line). "AGE:" and the space following it must be matched exactly. The period "." character matches any single character, and the asterisk (*) following it means "zero or more," so ".*" matches any number of characters up to the end of the line. Parentheses save the results of a match into numbered variables ( $\$ 1$ for the first set of parentheses, $\$ 2$ for the next, etc.). If there is a match, a message ("The age is:") and the value of $\$ 1$ are printed.

Finally, line 8 closes the input file handle. Because no more lines remain in the script, the interpreter will end after line 8 .

\section{EXAMPLE 2: SUMMARIZING DATA}

The program in Example 1 can be extended to not only report the subject's age, but also the mean of the three RTs. Here is one way to do it:

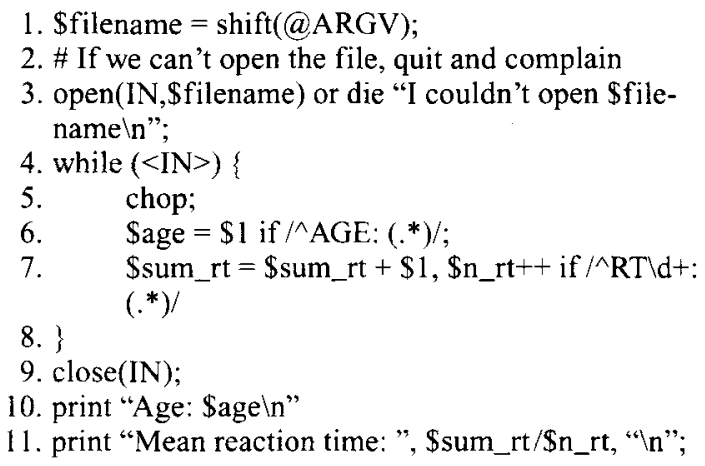

Lines 1-5 are the same as in Example 1: The program ascertains the filename, opens a file handle, and begins a loop to read in each line of the file, chopping off its newline character. In line 6 , a regular expression match is performed for AGE:, and if there is a match, $\$ 1$ (the text following AGE:) is assigned to the variable Sage.

In line 7 is another regular expression match. The caret again means that the match should begin at the beginning of the line. The " $\mathrm{d}$ " matches any digit, and 
the "+" following it means " 1 or more." So the expression is read as "at the beginning of the line, an 'RT' followed by one or more digits, followed by a colon and a space, and assign all remaining characters to \$1." If a line of the file matches this pattern, whatever follows the colon is added to the variable Ssum_rt (sum of RTs), and the variable $\$ n_{-}$rt (number of RTs) is incremented by 1 . An important feature used in this example is that perl implicitly assumes that scalar variables are equal to 0 when they are first used numerically; initialization is not necessary.

Once the whole file has been examined, line 10 prints the age stored in \$age, and line 11 prints the mean RT by dividing $\$$ sum_rt by $\$ n \_r t$.

\section{EXAMPLE 3: CODING VERBAL DATA}

The following program counts the number of times the subject refers to him- or herself in the protocol:

1. Sfilename $=\operatorname{shift}(@$ ARGV);

2. \# If we can't open the file, quit and complain

3. open(IN,\$filename) or die "I couldn't open \$filenameln";

4. while $(<\mathrm{IN}>)$ \}

5. chop;

6. last if $/ \wedge$ PROTOCOL/;

7. $\}$

8. while $(<\mathrm{IN}\rangle)\}$

9. $\quad$ Scount $=\$$ count $+\mathrm{s} / \mathrm{b}(\mathrm{I} \mid \mathrm{me}) \backslash \mathrm{b} / / \mathrm{g}$;

10. $\}$

11. close(IN);

12. if (\$count)

13. print "Subject used the words I or me \$count timesln";

14 \} else \{

15. print "Subject never used the words I or

16. me. In";

As before, the program opens the file and begins reading lines. The goal is to find the line at which the protocol begins. Lines 4-7 do this, reading in each line of the file until the PROTOCOL line is found and then leaving the loop. Line 6 is read as "this should be the last time through the loop if we match PROTOCOL."

Next, the program must read in each line and count the number of occurrences of "I" or " $m$.." The loop in lines 8-10 performs this feat. Line 9 uses the regular expression substitution operator, $s / /$. This operator matches the regular expression between the first and second slashes, and replaces it with the text between the second and third slashes. The " $\mathrm{g}$ " after the third slash instructs the operator to match and replace every occurrence in the line ("globally") instead of just the first one. In this case, the program looks for the regular expression " $b(\mathrm{I} \mid \mathrm{me}) \backslash \mathrm{b}$." The vertical bar is read as "or," and the "lb" matches a word boundary and keeps us from matching "smear" instead of "me." The regular expression is then "match ei- ther 'I' or 'me' as individual words." Each match is replaced by a blank string, and the "s///g" operator returns the number of replacements made, which is added to \$count.

Finally, in lines 12-16, the results are printed out. If $\$$ count is set (to anything but 0 ), \$count is reported; otherwise, it is reported that no matches were found.

\section{EXAMPLE 4: MEDLAN AGE FROM MULTIPLE DATA FILES}

A more complicated program might be given many filenames as arguments and collate the data from all of the files. Following is how we might report the median age of the subjects in a set of files:

1. foreach \$filename (@ARGV) \{

2. \# If we can't open the file, warn and go to the next

3. unless (open(IN,\$filename)) \{

4. warn "I couldn't open \$filename $\backslash n$ ";

$5 . \quad$ next;

6. $\quad\}$

7. while $(<\mathrm{IN}>)\}$

8. next unless /^AGE: $\left(.^{*}\right) /$;

9. $\quad$ push $(@$ ages, \$1);

$10 . \quad$ last;

11. \}

12. $\quad \operatorname{close}(\mathrm{IN})$

13. $\}$

14. @ages=sort $\{\$ a \Leftrightarrow \$ b\} @$ ages;

15. while (@ages $>2)\{$

16.1shift(@ages);

17. pop(@ages);

18.

19. \$median = \$ages $[0]$ if @ages $==1$;

20. $\$$ median $=(\$$ ages $[0]+\$ \operatorname{ages}[1]) / 2$ if @ages $==2$;

21. print "The median age is \$median $\backslash n$ ";

The outer "foreach" loop iterates over the filenames given as arguments. Lines 3-6 are read as "Unless I can open \$filename, warn the user of the problem and go on to the next file."

In lines $7-11$, the age is extracted from the currently open file by matching the regular expression. The age is "pushed" onto the end of the list @ages, which perl assumes is initially empty.

Once all the ages are on the list, line 14 sorts the list numerically (the exact details of the sort command are beyond the scope of this article). The loop in lines 15-18 is an intuitive if not very efficient way to find the median. When the list variable @ages is used in a context in which a scalar would be expected (such as (a)ages >2), it represents the number of elements in the list. As long as there are more than two elements, the user removes the first element (with shift()) and the last element (with pop()). When the loop ends, there will either be a single element left in the list (the median value if the list had an odd number of elements) or two elements left (if the list 
had an even number of elements). Lines 19-20 compute the median when one or two elements remain, respectively. ${ }^{2}$ Finally, line 21 prints the result.

As the example shows, lists in perl do not have to be predeclared as a specific length-lists grow and shrink as objects are added and removed. The same program would work for 5 files or 50,000 files.

\section{DISCUSSION}

Software tools should be appropriate to the tasks they are used for. $\mathrm{C}$ is a powerful programming language, but it is often unwieldy-hundreds of lines of $C$ may be necessary to accomplish even simple tasks. On the other hand, batch languages like SAS can be used to efficiently write programs to do standard data manipulations, but lack the flexibility to do more specialized operations. One of perl's great virtues is that it is often "just the right size" for problems that face psychologists every day-flexible enough to be adapted to any data file arrangement, powerful enough to do nearly anything with the data, and compact enough to do it painlessly.

Perl scripts are particularly useful as "glue" between other programming languages or software packages. For example, after data are collected from subjects using MEL Professional (Psychology Software Tools, Inc., 1997), perl scripts can be used to summarize and check the data, perform basic analyses, and reformat the files for input to a statistical software package. If the statistical software produces voluminous output, perl scripts can read in the results of the analyses and provide reports and summaries. The scripts can be written iteratively, as they are needed.

The examples given here illustrate only a fraction of perl's capabilities: Nothing has been said about subroutines, associative arrays, references, or objects, for example. Perl has a complete set of mathematical functions. A large number of user-written "modules" are available that provide additional functions ranging from approximate pattern matching ("match 'hippocampus' but allow up to 2 spelling errors") to operating a Web server. Perl's built-in debugger (itself written in perl) makes it easy to step through the execution of a perl program line by line.

Like any tool, perl has its limitations as well. As an interpreted language, it often runs more slowly than com- piled languages like $\mathrm{C}$ when performing calculations. And, although it is possible to implement a neural network in perl (indeed, a module exists for just that purpose), training the network is likely to require considerably more time than a network implemented in $\mathrm{C}$ or Pascal. Although perl is numerically capable, and some basic statistical modules are available, its current level of statistical ability is similar to that of FORTRAN or other general purpose languages-SAS would be a more appropriate tool for performing an analysis of variance or factor analysis.

For many problems, however, perl is an ideal programming language. Because it is free, platform independent, and designed for text processing, perl is an invaluable tool to the psychological researcher who works with computer data files.

\section{REFERENCES}

Psychology Software Tools, Inc. (1997). MEL Professional [Computer software]. Pittsburgh: Author.

SAS INSTITUTE (1996). SAS [Computer software]. Cary, NC: Author. SCHWARTZ, R. L., \& ChRistiansen, T. (1997). Learning perl (2nd ed.). Sebastopol, CA: O’Reilly \& Associates.

SRINIVASAN, S. (1997). Advanced perl programming. Sebastopol: O'Reilly \& Associates.

WALL, L. (1986). Perl [Computer programming language]. Pasadena, CA: Author.

Wall, L., Christiansen, T., \& Schwartz, R. L. (1996). Programming perl (2nd ed.). Sebastopol, CA: O'Reilly \& Associates.

\section{NOTES}

1. Perl users traditionally invent new and playful meanings for the acronym. Wall's is "pathologically eclectic rubbish lister."

2. A more efficient procedure for computing the median of a sorted list would be as follows:

\$list_length=@ages;

\# If there are an odd number of elements in the list \# (i.e., \$list_length modulo $2=1$ ), the middle element is \# (\$list_length - 1)/2. Ex: with 3 elements, $(3-1) / 2=1$

\$median $=$ \$ages $[($ \$list_length-1 $) / 2]$ if $\$$ list_length $\% 2==1$;

$\#$ If there are an even number of elements in the list, \# take the mean of the middle elements. Ex: with 4 elements, \# the two middle elements are $(4 / 2-1)=1$ and $(4 / 2)=2$. Smedian $=$

(\$ages[\$list_length/2 - 1] + \$ages[\$list_length/2])/2 if Slist_length $\% 2=0$;

(Manuscript received October 6, 1997; revision accepted for publication December 15, 1997.) 